\title{
Energy Minimized design of Mixed Line Rate IP-over-WDM Network
}

\author{
Digvijay Singh \\ Department of Electronics and Communication Engineering \\ Graphic Era University, Dehradun, Uttrakhand, India
}

\begin{abstract}
Power consumption in IP-over-WDM networks has become a major concern, more so due to rapidly evolving data intensive applications. Increasing power consumption also increases the environmental cost (carbon emission). Carbon emissions are the result of power generation using non-renewable (traditional hydrocarbons) energy resources. Usage of non-renewable energy resources (such as hydrocarbons, fossil fuel) is a major cause of global warming. An idea to reduce carbon emission is to maximize the use of renewable energy at each node .This not only gives the opportunity to reduce the carbon emission but also leads to development of sustainable infrastructure. Wind and solar are two important resources of renewable energy today. However, green energy generated by these resources is dependent on time of the day and environmental conditions. Since green energy supply is intermittent and not always available, green nodes are backed up by traditional hydrocarbon grid energy. Efficient use of green energy to minimize carbon emission and energy cost is a challenge. We propose MILP (mixed integer linear program) that minimizes the overall non-renewable energy consumption of the MLR WDM network. This MILP model uses mixed-line-rate (MLR) over the single-line-rate (SLR) in WDM. We also compare the non-renewable energy consumption of MLR-WDM network with that of SLR-WDM network. Optimization results indicate that proposed MILP can reduce the overall non-renewable energy consumption of the network up to $35 \%$. In addition, it also reduces the number of transponders used in MLR as compared to SLR networks. Since the number of transponders utilized in the optical layer is directly proportional to the power consumed, therefore with MLR network, non-renewable energy consumption can be reduced in comparison to SLR network.
\end{abstract}

Keywords- IP-over-WDM, SLR, MLR

\section{INTRODUCTION}

Telecommunication networks today are going through technological changes to support huge data traffic while controlling their energy consumption. Lot of research is being focused towards improving the over all energy efficiency of the network. The related work can be classified into three categories: energy-efficient network design, energy-aware traffic grooming and turning off selective network elements.

Energy-efficient network design: The authors in [1] propose an approach to minimize energy consumption of IP over WDM networks where energy consumption of IP routers, EDFAs and transponders is jointly minimized. The authors focus on energy consumption of the IP over WDM network with lightpath bypass whereby all the data traffic, whose destination is not the intermediate node, is bypassed via a cut-through light- path. In [2], authors develop mathematical models to design energy and cost efficient MLR optical networks. They investigated the energy costs of MLR and SLR networks. The results indicate that MLR (Mixed Line Rate) can improve the energy efficiency of the network under most traffic loads, whereas in case of longer physical links where transmission reaches of line rates are not feasible, certain SLR (Single Line Rate) networks can be a better option. In [3], a LP (Linear Program) optimization model is developed for hybrid power IP over WDM networks. Hybrid-power network is composed of an IP layer and optical layer. The power of this network is mixed being composed of non-renewable and renewable energy. In this paper, a heuristic has also been proposed to minimize the non-renewable energy 
consumption of the hybrid-power IP over WDM network. [4], describes the pros and cons of optical and electronic technologies in terms of energy efficiency at component and transmission levels. It compares the power dissipation of large-capacity optical and electronic cross-connects for a number of cross-connect architectures.

Energy-aware traffic grooming.In [5], the total power consumption of IP over WDM network is modeled in terms of power consumption of individual lightpaths. An ILP (Integer Linear Program) is formulated for energy- aware grooming problem. In [6], the authors propose an MILP and a heuristic to solve the RWA (routing and wavelength assignment) problem and decrease the number of lightpath interfaces to minimize the energy consumption of the network. The authors in [7], target a green provisioning strategy for optical backbone networks to reduce the operational power. Operational power is route and strategy dependent e.g. optical bypass vs. traffic grooming). An auxiliary-graph based model is proposed to identify the energy consumed by the operation. Results shows that energy-aware traffic grooming needs the least operational power under various scenarios, compared to a direct-lightpath and a traffic-grooming approach. In [8] and [9], the authors focus on the energy aware dynamic traffic grooming in optical networks, based on the methodology of auxiliary graph. The authors minimize energy consumption of devices in the network on the basis of traffic profile variations during different hours of the day. In [10], authors present a heuristic approach, the robust and integrated grooming for power and port cost efficient design in live IP over WDM networks. The authors integrate the advantages of hybrid grooming (i.e. traffic grooming along with optical bypass) and waveband merging to achieve higher power efficiency.

Turning off selective network elements: The authors in [11] propose a scheme to reduce energy consumption by turning off idle line cards and chassis of routes based on the traffic variation during the time of the day. It enables carriers to design energy-efficient networks by dynamically reconfiguring network devices, ensuring a minimal impact on the live traffic served by the network. In [12], a scheme is proposed to shut down idle line cards (and corresponding optical circuits associated with the line cards) in low demand hours. Similarly in [13], the authors have also proposed to save energy by shutting down idle line cards and chassis, of IP over WDM when the traffic load is low and minimizing the potential traffic interruption when the line cards and chassis are shut down. The authors in [14], also propose an approach to selectively switch off optical links. The key contribution of this paper is to minimize the energy consumption of the whole IP over WDM MLR 14 node network on the basis of total renewable energy available at nodes using an MILP model. The difference of this study from the existing work [15] which proposes a MILP model for a SLR IP over WDM network while our paper proposes a MILP model for a MLR network. The rest of the paper is organized as follows. In Section II, we present the system model and renewable energy calculations. Section III presents MILP optimization model. Result and discussion are presented in section IV. The paper is concluded in section $\mathrm{V}$.

\section{SYSTEM MODEL FOR IP-OVER-WDM MLR NETWORK}

\section{A. System Model-}

This paper focuses on greening the IP over WDM network model. Since assuring the availability of sufficient renewable energy at each node location is difficult to estimate due to intermittent nature of renewable energy resources. Energy output from these resources depends on local weather conditions which is susceptible to change. Data traffic consumption pattern varies throughout the day. Our model relies on the availability of renewable energy throughout the day but due to intermittent output from these energy resources, this model also considers the network nodes to be backed up by non renewable energy resources (i.e. energy conventional from utility grid) for the proper operation of network nodes under assumed energy constraints. We propose a transparent IP over WDM MLR model. WDM combines and transmits multiple optical signals on the same fiber simultaneously. IP over WDM network is the packet data service over the optical fiber network. The main advantage of this network is high data transmission speed e.g. 10/40/100 Gbps and its interoperability among various prevailing standards like ATM, SONET/SDH and Ethernet. 


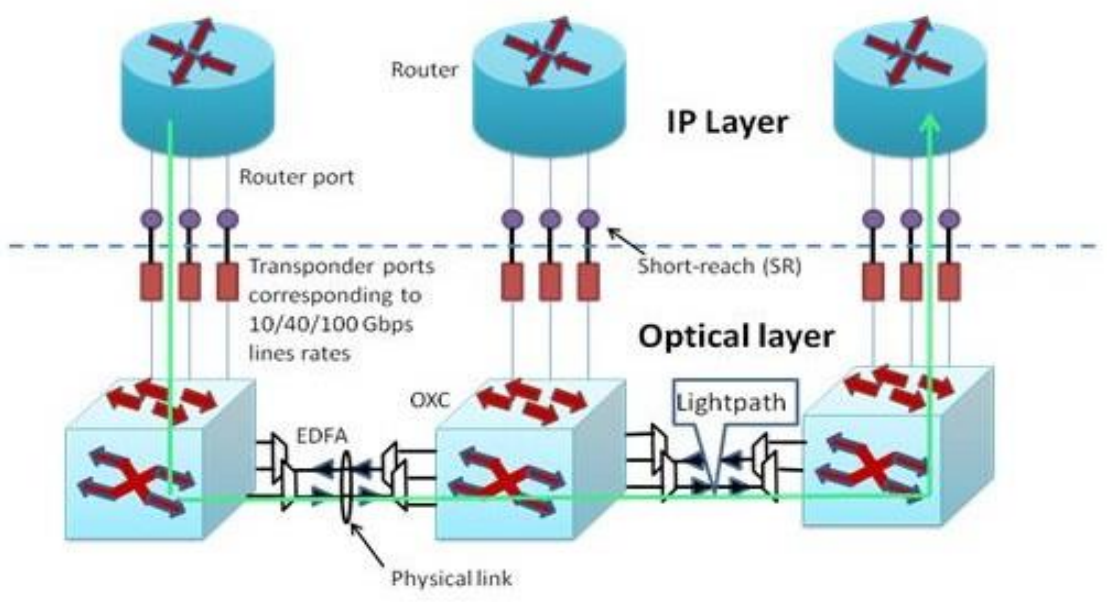

Figure 1. IP over WDM MLR Network Model

WDM networks can be classified on the basis of transponders used in the network as transparent, translucent and opaque. Transponders or $3 \mathrm{R}$ units (reamplification, reshaping and retiming) in an optical transport network (OTN) are required for O-E-O conversion at intermediate network nodes. In opaque network, O-E-O conversion is performed at each intermediate node, as this approach provides flexibility in terms of network management, design and control but on the other hand it increases the overall cost and energy consumption of the network [10]. Transparent network utilize the optical bypass approach in which optical signals bypass the intermediate or regenerative nodes in the network. In translucent networks both the features of the network i.e. opaque and transparent are used simultaneously. For our energy efficient MILP model we have used MLR for lightpaths instead of SLR as in conventional networks. Also the network nodes in the network are backed up by conventional non renewable energy resource in case there is not sufficient renewable energy available on a day, network can be operated uninterruptedly. IP traffic over WDM is suitable choice for ever increasing data traffic in internet networks. IP routers are the elements of IP layer. OXC and transponders are the network element of optical layer. OXC is a network element used for switching high speed signals in telecommunication network. We have used transparent OXC in our model. Transparent OXC performs switching action on high speed optical signals and transponders are responsible for O-E-O conversion of signals in the network. Short Reach (SR) interface provides interoperability between optical equipments from various vendors. Erbium Doped Fiber Amplifiers (EDFAs) are used to boost the strength of optical signals propagating through optical fiber. Nodes are connected by optical fiber to form physical topology. Any two IP routers will be connected by all optical WDM channel called lightpaths.

Table1 Optical reach, normalized transponder energy consumption and normalized cost for 10/40/100G

\begin{tabular}{cccc}
\hline $\begin{array}{c}\text { Rate } \\
\text { (Gbps) }\end{array}$ & $\begin{array}{c}\text { Optical reach } \\
(\mathrm{kms})\end{array}$ & $\begin{array}{c}\text { Energy } \\
\text { consumption }\end{array}$ & $\begin{array}{c}\text { Transponder } \\
\text { cost }\end{array}$ \\
\hline 10 & 3000 & $1 \mathrm{X}$ & $1 \mathrm{X}$ \\
40 & 2000 & $2.6 \mathrm{X}$ & $2.5 \mathrm{X}$ \\
100 & 1500 & $5.7 \mathrm{X}$ & $3.75 \mathrm{X}$ \\
\hline
\end{tabular}




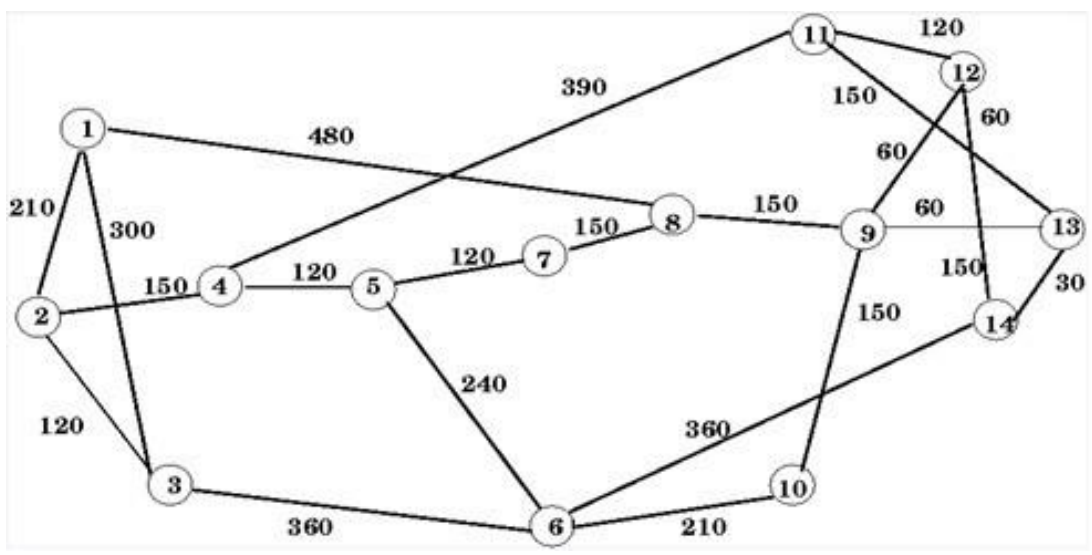

Figure 2. 14-Node NSFNET topology

The node set corresponds to IP routers and OXCs. Within a single node, an IP router is connected to an OXC via SR interfaces. The link set consists of the physical fiber links in the network.

MLR is the combination of heterogeneous wavelength capacity rates that can be used to carry traffic in a network. As compared to SLR networks which use a single wavelength channel capacity, MLR offers (10/40/100 Gbps) capacities to serve the traffic [11][12]. The basic idea behind MLR is to assign line rates to lightpaths such that optical reach of line rates supports maximum route length of path. MLR enables efficient data transmission in terms of number of transponders utilized, energy consumption and residual capacity. In this paper we are considering the energy consumption of transponders associated to channel rate (i.e. 10/40/100Gbps) and also the following values of optical reach as shown in Table 1. For SLR we have considered lightpaths capacity as 40 Gbps. Table 2 shows the energy consumption values calculated for transmission of 44 Gbps of data through MLR/SLR network. With SLR network where only 40Gbps lightpaths can be set up, we need two such lightpaths to carry the required traffic. However with MLR network we can set up one 10Gbps and one 40Gbps lightpaths to carry the required traffic. The energy consumption is less for MLR as shown Table 2. Moreover the residual capacity is $36 \mathrm{Gpbs}$ in SLR network, so this lightpath will have to drop down at intermediate router ports to collect more traffic for efficient utilization, thereby increasing the delay in the network. On the other hand, residual capacity is $6 \mathrm{Gbps}$ in MLR as it utilizes lightpath more efficiently.

Table 2 Energy consumption for transmission of 44 Gbps of data through MLR/SLR network.

\begin{tabular}{ccccc}
\hline Rate (Gbps) & $\begin{array}{c}\text { Traffic demand } \\
\text { (Gbps) }\end{array}$ & $\begin{array}{c}\text { Transponders } \\
\text { used }\end{array}$ & $\begin{array}{c}\text { Energy } \\
\text { consumed }\end{array}$ & $\begin{array}{c}\text { Residual capacity } \\
\text { (Gbps) }\end{array}$ \\
\hline SLR (40) & 44 & $2 * 40$ & $2.6 * 2=5.2$ & 36 \\
MLR & 44 & $1 * 10+1 * 40$ & $1+2.6=3.6$ & 6 \\
$(10 / 40 / 100)$ & & & & \\
\hline
\end{tabular}


Table 3 Renewable energy $(\mathrm{kWh})$ at network nodes from May $1^{\text {st }}$ to May $7^{\text {th }}$

\begin{tabular}{cccccccc}
\hline Node & May1 & May2 & May3 & May4 & May5 & May6 & May7 \\
\hline 1 & 156.8 & 100.0 & 150.0 & 105.6 & 100.3 & 138.5 & 203.3 \\
2 & 248.1 & 200.5 & 150.7 & 145.7 & 240.2 & 250.3 & 141.2 \\
3 & 172.4 & 120.6 & 200.7 & 110.7 & 165.3 & 176.2 & 176.5 \\
4 & 177.4 & 120.5 & 120.8 & 251.0 & 160.5 & 159.2 & 160.5 \\
5 & 271.8 & 258.7 & 110.7 & 110.3 & 257.2 & 260.2 & 180.7 \\
6 & 269.4 & 110.4 & 100.3 & 165.4 & 265.5 & 254.2 & 200.5 \\
7 & 132.1 & 134.9 & 180.4 & 150.0 & 110.4 & 251.2 & 200.5 \\
8 & 29.10 & 260.3 & 127.6 & 180.1 & 180.2 & 194.7 & 256.4 \\
9 & 276.3 & 140.5 & 101.2 & 170.2 & 170.2 & 163.7 & 185.3 \\
10 & 169.2 & 105.3 & 220.5 & 250.2 & 260.2 & 250.1 & 191.2 \\
11 & 173.2 & 240.2 & 230.6 & 160.3 & 250.3 & 241.2 & 210.3 \\
12 & 235.8 & 270.1 & 200.4 & 220.3 & 230.7 & 237.2 & 200.4 \\
13 & 297.3 & 150.2 & 191.2 & 180.4 & 207.7 & 198.4 & 292.3 \\
14 & 184.4 & 261.3 & 170.1 & 286.0 & 170.5 & 241.5 & 274.4 \\
\hline
\end{tabular}

\section{B. Renewable Energy calculations-}

For the network, total renewable energy at a node is the sum of solar and wind energy available at a node in a day.Output of $1 \mathrm{sq}$ meter of silicon solar cell for a day is $0.28 \mathrm{~kW}$ [14]. Area of silicon solar panels configured at each node is $100 \mathrm{sq}$ meter. Assuming that solar plants can work 12 hours a day and wind turbines operate all day i.e. 24 hours [17].Solar energy harvested in a day is given as:

$P_{S}=0.28 \times(1-$ sky cover $) \times 12 \times 100$

For sky cover of $75 \% P_{S}$ obtained is $84 \mathrm{kWh}$. Wind power harvested in a day is given as:

$P_{W}=0.5 \alpha A \rho v^{3}$

where $\alpha$ is efficiency factor considered to be $0.5, A=200$ is effective windward area and $\rho=1.3 \mathrm{Kg} / \mathrm{m}$ is density of air and $v=3.6 \mathrm{Km} / \mathrm{hour}$ is wind speed .For wind turbines operating for 24 hours a day $P_{W}$ is $72.8 \mathrm{kWh}$

Total renewable energy $\left(P_{S}+P_{W}\right)$ harvested in a day is $156.8 \mathrm{kWh}$.

Renewable energy values at NSFNET nodes from May 1st to 7 th are shown in Table 3.

\section{MILP OPTIMIZATION MODEL FOR MINIMIZING NON-RENEWABLE ENERGY CONSUMPTION IN MLR NETWORK}

Our objective is to minimize non renewable energy consumption in IP over WDM network in a day.

A. Input parameters for $L P$ -

- $\mathrm{G}=(\mathrm{N}, \mathrm{E})$ : physical topology consists of a set of nodes $\mathrm{N}$ and $\mathrm{E}$ links

$-f^{s d}$ : traffic matrix in Gbps

- $E_{t r i}$ amount of (estimated) renewable energy (sum of solar and wind energy) available in a day at node $i$

- $r=r_{1}, r_{2} \ldots r_{k}$ : set of available lightpath rates in Gbps

- $\Delta_{\mathrm{i}}=$ number of transponders at a node $i$

$B$. Varaibles for the $L P$ -

- $f_{i j}^{g d}:$ traffic from source $s$ to destination $d$ routed between node $i$ and $j$

- $f_{i j}$ : total traffic between node $i, j$

- $v_{i j r}$ : number of lightpaths of rate $\mathrm{r}$ between virtual link $i, j$

- $N R E_{i}$ : non-renewable energy consumption at node $i$ in a day

$-\nabla_{i r}=$ number of transponders at node $i$ of rate $r$ 


\section{Problem Formulation-}

Objective: Minimize $\sum_{\mathrm{i}} N E R_{\mathrm{i}}$

Subject to: Flow conservation constraint in the IP layer. Traffic between s-d pair is allowed to be spilt and transmitted over multiple flow paths.

$\sum_{j} f_{i j}^{g d}-\Sigma_{j} f_{i j}^{g d}=\left\{\begin{array}{c}f^{s d}, \text { if } s=i \\ -f^{v d}, \text { if } d=i \text { for all } s, d, i \\ 0, \text { otherwise }\end{array}\right.$

Traffic flows in IP layer are bidirectional and are co-routed

$f_{i j}^{g d}=f_{i j}^{g d}$ for all $i_{i} j, s_{,} d$

Virtual lightpath links 10/40/100 Gbps have sufficient capacity to carry these IP traffic flows

$\sum_{s \in \mathbb{N}} \sum_{d \in N} f_{i j}^{g d} \leq \sum_{r=1}^{a} r v_{i j r}$ for all $i_{i} j$

Lightpath virtual links for the optical layer are bi-directional

$v_{i j r}=v_{j i r}$ for all $i_{i} j, r$

Unidirectional lightpaths having capacities (i.e. 10/40/100 Gbps) starting from node $i$ is equal to the total number of transponders at node $i$.

$\sum_{j} v_{j i r}=\nabla_{i r}$ for all $i, r$

Router ports/transponder deployed at each node does not exceed the maximum number set in this study

$\sum_{y=1}^{\mathrm{a}} \nabla_{\mathrm{ir}} \leq \Delta_{\mathrm{i}}$ for all $i$

Renewable energy and non-renewable energy at each node can satisfy the total energy consumption of the node

$N E R_{i}+R E_{i} \geq \sum_{r=1}^{3} \Delta_{i r}\left(E_{t r i}\right)$

\section{RESULTS AND DISCUSSION}

To solve our MILP problem, we have used IBM ILOG CPLEX software on an Intel core i5 machine with a 4Gb RAM. To simplify our optimization problem, we have used the amount of renewable energy present at various geographical nodes but ignored the geographical distribution and location of network nodes. Further, energy consumption due to OXCs, SR and EDFAs has not been taken into account as these network elements have relatively small contribution in the total energy consumption of the network. Total renewable and non-renewable energy available in the network is the summation of renewable and non-renewable energy present at each node. Traffic demand between each node pair is generated by random function uniformly distributed over range [10, $2 \mathrm{X}$ 10 ], where $X$ is average demand intensity of the network.

For NSFNET network topology and the values of optical reach as in Table $1100 \%$ of connections can be established for $10 \mathrm{Gbps}$ line rate, $70 \%$ of connections can be established for $40 \mathrm{Gbps}$ line rate and $40 \%$ connections can be established for $100 \mathrm{Gbps}$ line rate.

Fig. 3 shows the non-renewable energy consumption (kWh) in MLR and SLR networks from May 1st to May 7th for average traffic demand intensity of $60 \mathrm{Gbps}$, i.e., $\mathrm{X}$ is 60 and nodes pairs have traffic demand over the range $[10,110]$ Gpbs. Renewable energy calculations provide the total renewable energy available at nodes as shown in Table 3. Average value of renewable energy available at a node on May 1st and May 3rd is $206 \mathrm{kWh}$ and $160 \mathrm{kWh}$ respectively. Renewable energy available on May 1st is more compared to energy available on May 3rd. Therefore on May 1st total non-renewable energy consumption of the network is less than May 3rd. Furthermore it can be seen by employing MLR in network the total non-renewable energy consumption can be brought down compared to SLR network. 
Fig. 4 compares the percentage of non renewable energy consumption in MLR and SLR from May 1st to May 7th. MLR network achieves higher percentages of non-renewable energy saving consumption compared to SLR Network. This is because of the volume discount offered by the high-line- rates over low-line-rates.

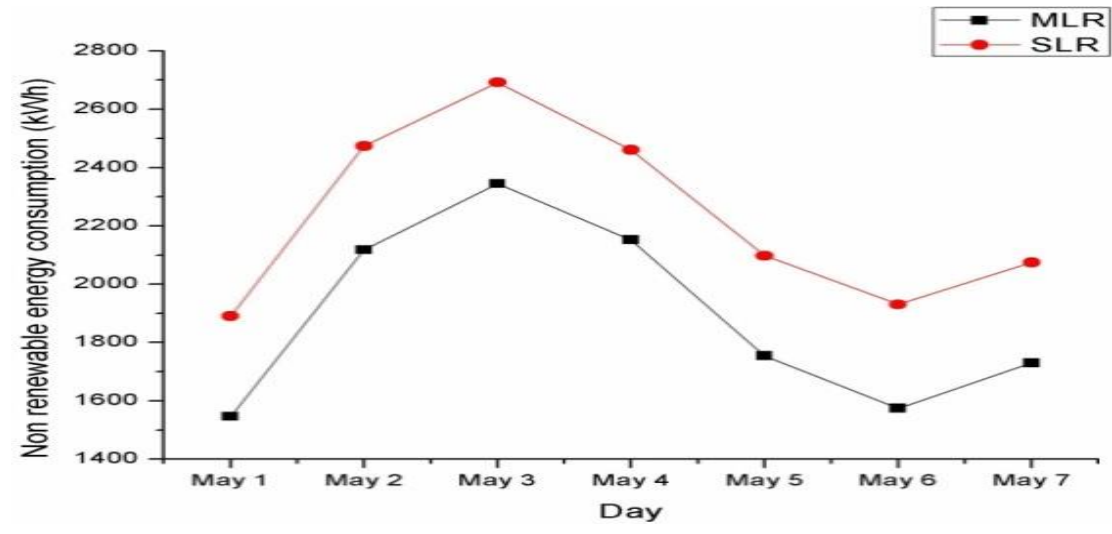

Figure 3. Non-renewable energy consumption (kWh) in MLR and SLR Network

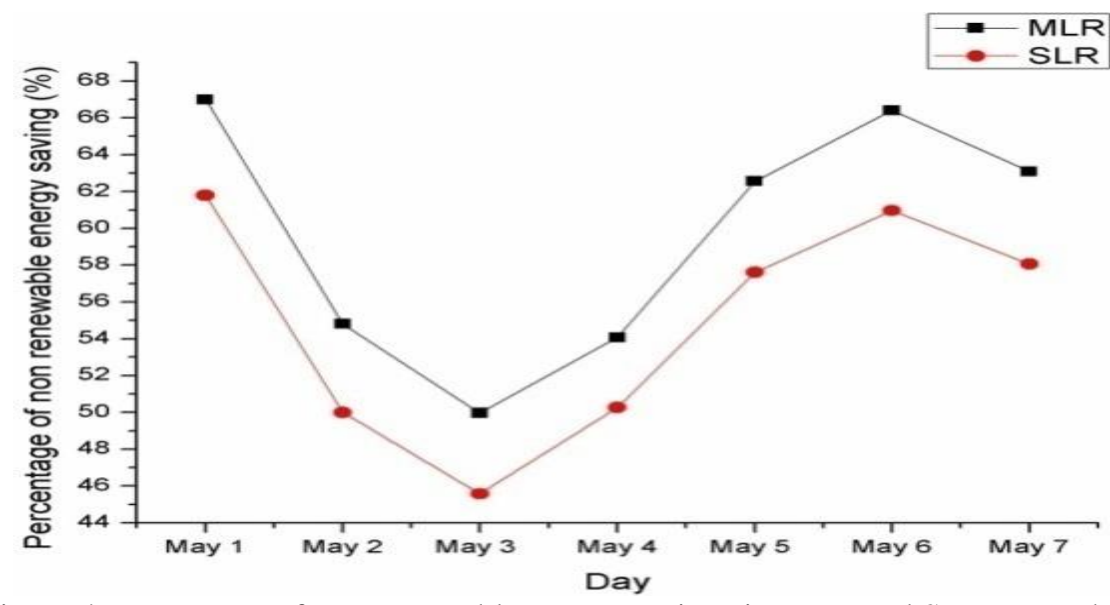

Figure 4. Percentage of non-renewable energy savings in MLR and SLR network

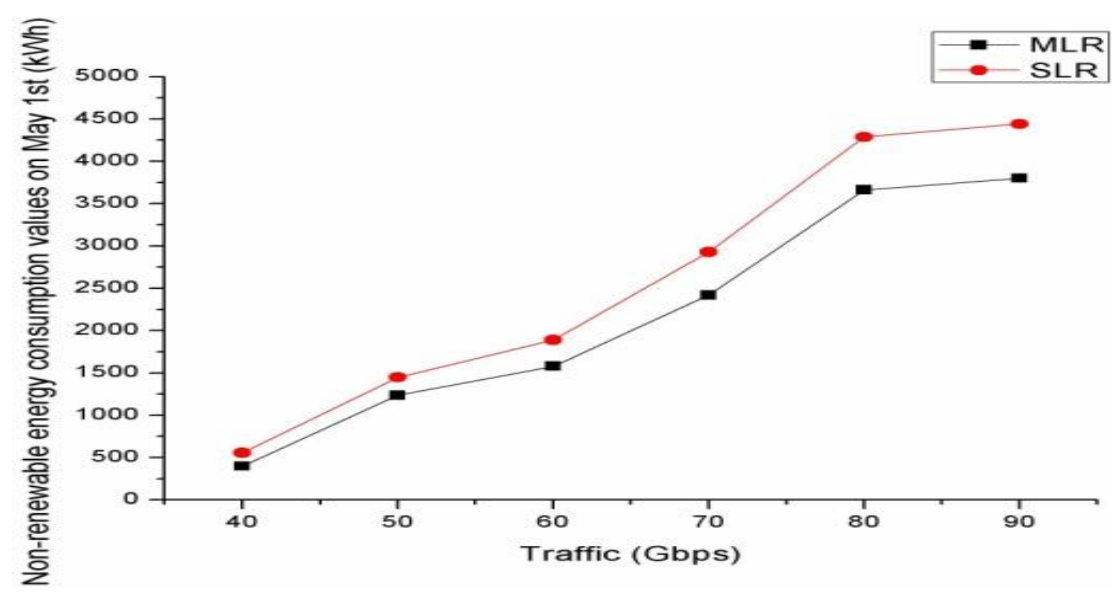

Figure 5. Non-renewable energy consumption values on May $1^{\text {st }}$ for MLR and SLR Network with varying traffic intensity 
Fig. 5 shows the non-renewable energy consumption values for MLR and SLR on May 1 st for varying average traffic intensity in the network. MLR has an advantage over SLR in terms of non-renewable energy consumption more so when the average traffic intensity in the network is high. Table 4 gives typical value of the number of transponders utilized by SLR and MLR network on May 1st for a varying traffic from 1 Tbps to 5 Tbps. As the traffic increases, SLR network consumes more transponder as compared to MLR network to carry data traffic.

Table 4 Number to transponder used in SLR and MLR network on May $1^{s t}$

\begin{tabular}{ccrrc}
\hline $\begin{array}{c}\text { Traffic } \\
\text { (Tbps) }\end{array}$ & SLR & & $\begin{array}{r}\text { MLR } \\
\text { 40G }\end{array}$ & $100 \mathrm{G}$ \\
\hline $1 \mathrm{~T}$ & 322 & 6 & 62 & 120 \\
$2 \mathrm{~T}$ & 658 & 44 & 168 & 200 \\
$3 \mathrm{~T}$ & 980 & 304 & 0 & 364 \\
$4 \mathrm{~T}$ & 1302 & 4 & 2 & 530 \\
$5 \mathrm{~T}$ & 1526 & 234 & 102 & 546 \\
\hline
\end{tabular}

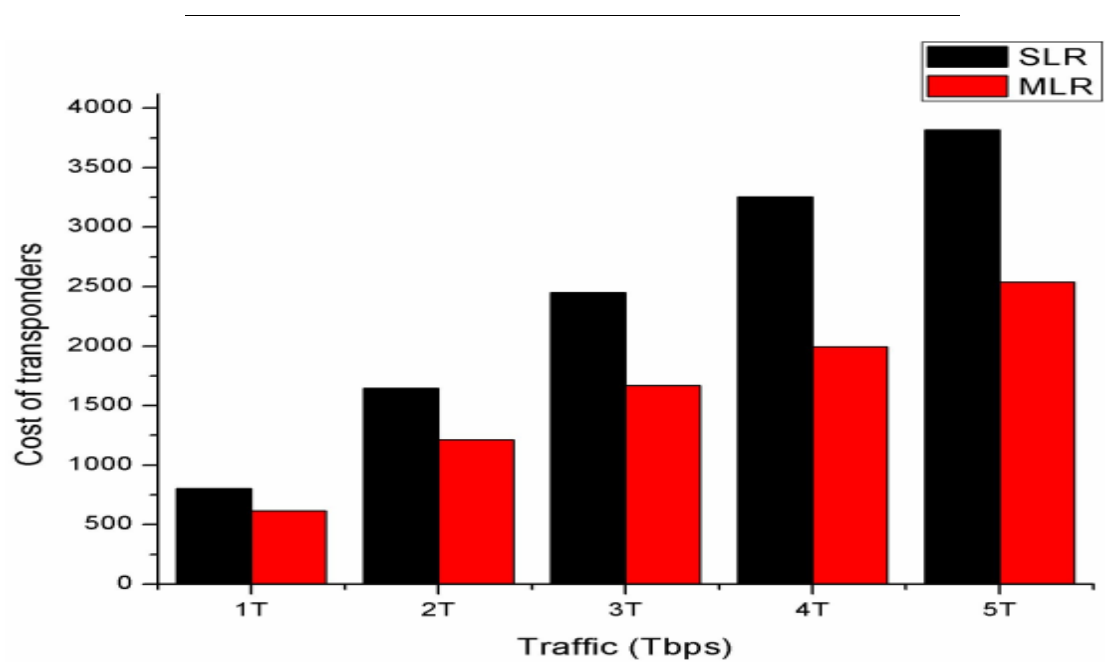

Figure 6. Cost of transponders used in SLR and MLR network on May 1st normalized wrt 10G transponder cost

Fig. 6 shows the cost of transponders used in SLR and MLR network on May 1st. Cost of transponders is directly proportional to number of transponder used in the network. Since SLR network consumes more number of transponders, therefore the cost of transponders used in SLR network is significantly higher than cost of transponders used in MLR network due to volume discount.

\section{CONCLUSION}

We developed an MILP model to minimize the overall energy consumption of the IP over WDM MLR network by considering the availability of total renewable energy at each network node. Optimization results show that proposed MILP model can reduce the overall non renewable energy consumption up to $35 \%$ or more as compared to conventional SLR networks powered by non renewable energy resources. Also MLR network provide efficient network operation as it requires less transponders and hence minimize the cost of the network. In this paper, we have only considered the transparent IP over WDM network to study the optimization results. However, opaque and translucent networks can also be analyzed to give a more practical view of today's IP-over-WDM network.

\section{REFERENCES}

1. Shen, Gangxiang, and Rodney S. Tucker. "Energy minimized design for IP over WDM networks." Optical Communications and Networking, IEEE/OSA Journal of 1.1 (2009): 176-186.

2. Chowdhury, Pulak, et al. "On the design of energy-efficient mixed-line-rate (MLR) optical networks." Lightwave Technology, Journal of 30.1 (2012): 130-139.

3. Dong, Xiaowen, Taisir El-Gorashi, and Jaafar MH Elmirghani. "IP over WDM networks employing renewable energy sources." Journal of Lightwave Technology 29.1 (2011): 3-14. 
4. Tucker, Rodney S. "The role of optics and electronics in high-capacity routers." Journal of Lightwave Technology 24.12 (2006): 4655-4673.

5. Yetginer, Emre, and George N. Rouskas. "Power efficient traffic grooming in optical WDM networks." Global Telecommunications Conference, 2009. GLOBECOM2009. IEEE. IEEE, 2009.

6. Huang, Shu, Deepa Seshadri, and Rudra Dutta. "Traffic grooming: a changing role in green optical networks." Global Telecommunications Conference, 2009. GLOBECOM 2009. IEEE. IEEE, 2009.

7. Xia, Ming, et al. "Green provisioning for optical WDM networks." Selected Topics in Quantum Electronics, IEEE Journal of 17.2 (2011): 437-445.

8. Hasan, Mohammad M., Farid Farahmand, and Jason P. Jue. "Energy-awareness in dynamic traffic grooming." Optical Fiber Communication Conference. Optical Society of America, 2010.

9. Hasan, M. Masud, et al. "Traffic grooming in green optical networks." Communications (ICC), 2010 IEEE Inter- national Conference on. IEEE, 2010.

10. Hou, Weigang, Lei Guo, and Xuetao Wei. "Robust and integrated grooming for power-and port-cost-efficient de- sign in IP over WDM networks." Lightwave Technology, Journal of 29.20 (2011): 3035-3047.

11. Zhang, Yi, et al. "Energy optimization in IP-over-WDM networks." Optical Switching and Networking 8.3 (2011): 171-180.

12. Idzikowski, Filip, et al. "Saving energy in IP-over-WDM networks by switching off line cards in lowdemand scenarios." Optical Network Design and Modeling (ONDM), 2010 14th Conference on. IEEE, 2010.

13. Zhang, Yi, et al. "Time-aware energy conservation in IP- over-WDM networks." Photonics in Switching. Optical Society of America, 2010.

14. Coiro, Angelo, et al. "Reducing power consumption in wavelength routed networks by selective switch off of optical links." Selected Topics in Quantum Electronics, IEEE Journal of 17.2 (2011): 428436.

15. Gattulli, Mirko, et al. "Low-emissions routing for cloud computing in IP-over-WDM Networks with data centers." Selected Areas in Communications, IEEE Journal on 32.1 (2014): 28-38. 